\title{
Single-Particle Diffraction with the X-Ray Free Electron Laser: New Opportunities to Study Structure and Function in Biology
}

\author{
Peter Schwander \\ University of Wisconsin, Milwaukee, WI 53211, USA \\ pschwan@uwm.edu
}

The X-Ray Free Electron Laser (XFEL) enables diffraction from individual biomolecules. The obvious advantage is that no crystals are required. But the true potential of single-particle diffraction unfolds in the presence of structural variability, intrinsic to biological function. Data analysis using manifold-based machine learning reveals the concerted structural changes and enables mapping the conformational spectrum, energy landscapes and compiling molecular movies. Performing singleparticle diffraction in a time-resolved manner can further advance these salient opportunities. 